\title{
Primera cita de nóctulo mediano (Nyctalus noctula) en Aragón (NE de España)
}

\author{
Luís Lorente Villanueva ${ }^{1 *}$, Carlos Llana UGalde ${ }^{2}$, José Manuel SÁnchez Sanz ${ }^{3}$
}

\author{
${ }^{1}$ Avda. Puente del Pilar 23, 6º B, 50014, Zaragoza \\ ${ }^{2}$ Conservación de la Biodiversidad, Servicio Provincial de Zaragoza, Departamento de Agricultura, \\ Ganadería y Medio Ambiente, $\mathrm{P}^{\mathrm{o}}$ María Agustín 36, 50071, Zaragoza \\ ${ }^{3} \mathrm{C} /$ Avda. de Movera ${ }^{\circ}$ 483, casa 5, 50194, Zaragoza
}

*Corresponding author e-mail: luislorentevillanueva@gmail.com

DOI: http://dx.doi.org/10.14709/BarbJ.7.1.2014.04

English title: First record of Noctule Bat (Nyctalus noctula) in Aragón (NE Spain)

\begin{abstract}
Resumen
Cuatro adultos de nóctulo mediano (Nyctalus noctula) fueron capturados recientemente en un estanque del Monasterio de Piedra (Nuévalos, Aragón, NE de España). Estas citas constituyen los primeros datos de su presencia en la Comunidad Autónoma de Aragón y uno de los pocos lugares conocidos con agrupaciones de esta especie en la Península Ibérica.
\end{abstract}

Palabras clave: Nyctalus noctula, primera cita, Aragón, España.

\begin{abstract}
Four adults Noctule Bat (Nyctalus noctula) were recently netted over a pond in the Monasterio de Piedra (Nuévalos, Aragón, NE Spain). These are the first unambiguous catches of this species in the Autonomous Community of Aragón. Besides, this site is one of the few localities in the Iberian Peninsula with groups of this bat species.
\end{abstract}

Key words: Nyctalus noctula, first record, Aragón, Spain.

\section{INTRODUCCIÓN}

El nóctulo mediano Nyctalus noctula (Schreber, 1774) tiene una distribución Paleártica, extendiéndose desde la Península Ibérica hasta Asia Central, y entre el sur de Escandinavia y quizás el norte de África (Simmons 2005). Es una especie migratoria que normalmente forma las colonias de cría en el centro y norte de Europa, y en algunas localidades aisladas en el sur (Ruedi et al. 1998; Dietz et al. 2009). En Europa, numerosas hembras migran hacia el norte para criar, $\mathrm{y}$ entre finales del verano y principios de otoño vuelven a las áreas meridionales de apareamiento e hibernación (Dietz et al. 2009; Alcalde 1999). Los territorios de verano pueden estar alejados más de $1000 \mathrm{~km}$ de los de invierno (Hutterer et al. 2005).

En España es una especie rara y muy localizada. Se tiene conocimiento de una colonia de cría en Aranjuez (Ruedi et al. 1998) y de otras tres colonias en Navarra (dos) y Lleida (una) con machos sedentarios durante gran parte del año, y con hembras que acuden para aparearse e hibernar, permaneciendo desde finales de agosto hasta marzo o mayo (Alcalde 1999; Camprodon \& Guixé 2013).

Los resultados de este artículo se han obtenido en el marco de un inventario de los murciélagos que se está realizando en el Monasterio de Piedra (Nuévalos, SO Zaragoza), y que está declarado Conjunto de Interés Cultural en la categoría de Jardín Histórico.

\section{MATERIAL Y MÉTODOS}

Para la captura de los murciélagos se emplearon tres redes finas de $9 \mathrm{~m}$ (Ecotone, Polonia), que fueron colocadas simultáneamente durante cinco noches, dos en otoño de 
2012, y tres en verano de 2013, sobre un estanque situado en el interior de los jardines del Monasterio de Piedra a $750 \mathrm{~m}$ de altitud (Datum: ETRS89, Huso UTM: 30T X: 602200, Y: 4560740). El entorno del punto de captura está formado por una alineación de bosque de ribera con árboles de especies autóctonas y por varios rodales con árboles ornamentales, entre los que destacan casi un centenar de plátanos de sombra (Platanus hybridus) de gran porte, plantados a finales del siglo XIX. Rodeando este enclave forestal de fondo de valle fluvial se extienden amplias zonas despejadas sobre sustrato calizo cubiertas de matorral mediterráneo y cultivos de secano en cotas superiores.

\section{RESULTADOS Y DISCUSIÓN}

En las dos jornadas de trampeo realizadas a mediados del mes de octubre de 2012 no fue capturado ningún nóctulo. Se repitieron otras dos sesiones en julio y otra en septiembre de 2013. El 18 de julio de 2013 fue capturado un macho adulto de nóctulo mediano, que fue identificado por la longitud del antebrazo, (antebrazo: 53,64 mm, 36,1 g), la morfología del trago y las alas (Dietz et al. 2009). Posteriormente, el 20 de septiembre de 2013 se capturaron dos hembras adultas (antebrazo y peso: $52,53 \mathrm{~mm}, 31,3 \mathrm{~g} ; 54,86 \mathrm{~mm}, 36,3 \mathrm{~g}$ ) y un macho adulto $(52,36 \mathrm{~mm}, 33,3 \mathrm{~g})$ sexualmente activo con glándula bucal característica y testículos escrotales. La Estación Biológica de Doñana (CSIC), confirmó la identificación de todos los individuos mediante análisis molecular de tejido alar obtenido con un "biopunch".
$N$. noctula ha sido citado previamente en Aragón por Woutersen \& Bafaluy Zoriguel (2001) pero la cita se considera dudosa al carecer de datos biométricos o genéticos que la avale (Alcalde 2008). Por ello, puede considerarse que ésta es la primera cita fiable de esta especie en Aragón.

La abundancia de $N$. noctula en Europa contrasta con su rareza en la Península Ibérica (Ibáñez et al. 1992). La cita que se aporta se suma a las pocas y dispersas que se han recopilado, y contribuye a mejorar el conocimiento que se tiene de las agrupaciones conocidas de la especie en España (Fig.1). Se observa una distribución claramente septentrional (Alcalde 2007), y en cierta medida existe cierta agregación espacial, considerando que tres de las cuatro provincias donde se han localizado las agrupaciones son adyacentes.

Los datos obtenidos sugieren la presencia de una agrupación de Nyctalus noctula en el Monasterio de Piedra, que podría mostrar el mismo patrón observado en otras colonias (Alcalde 2008; Camprodon \& Guixé 2013). Es decir, que ésta podría estar formada por una colonia de machos sedentarios, a la cual se unirían las hembras, y probablemente otros machos, desde finales del verano hasta la primavera, coincidiendo con el apareamiento y la hibernación.

El nóctulo mediano es una especie arborícola que acostumbra a refugiarse en huecos naturales de frondosas. La presencia en el Monasterio de Piedra de viejos plataneros centenarios en los que abundan orificios y superan en su mayoría los $25 \mathrm{~m}$ de altura, parece estar relacionada con la presencia de la especie. Una inspección superficial permitió comprobar la presencia de varios refugios potenciales, a veces

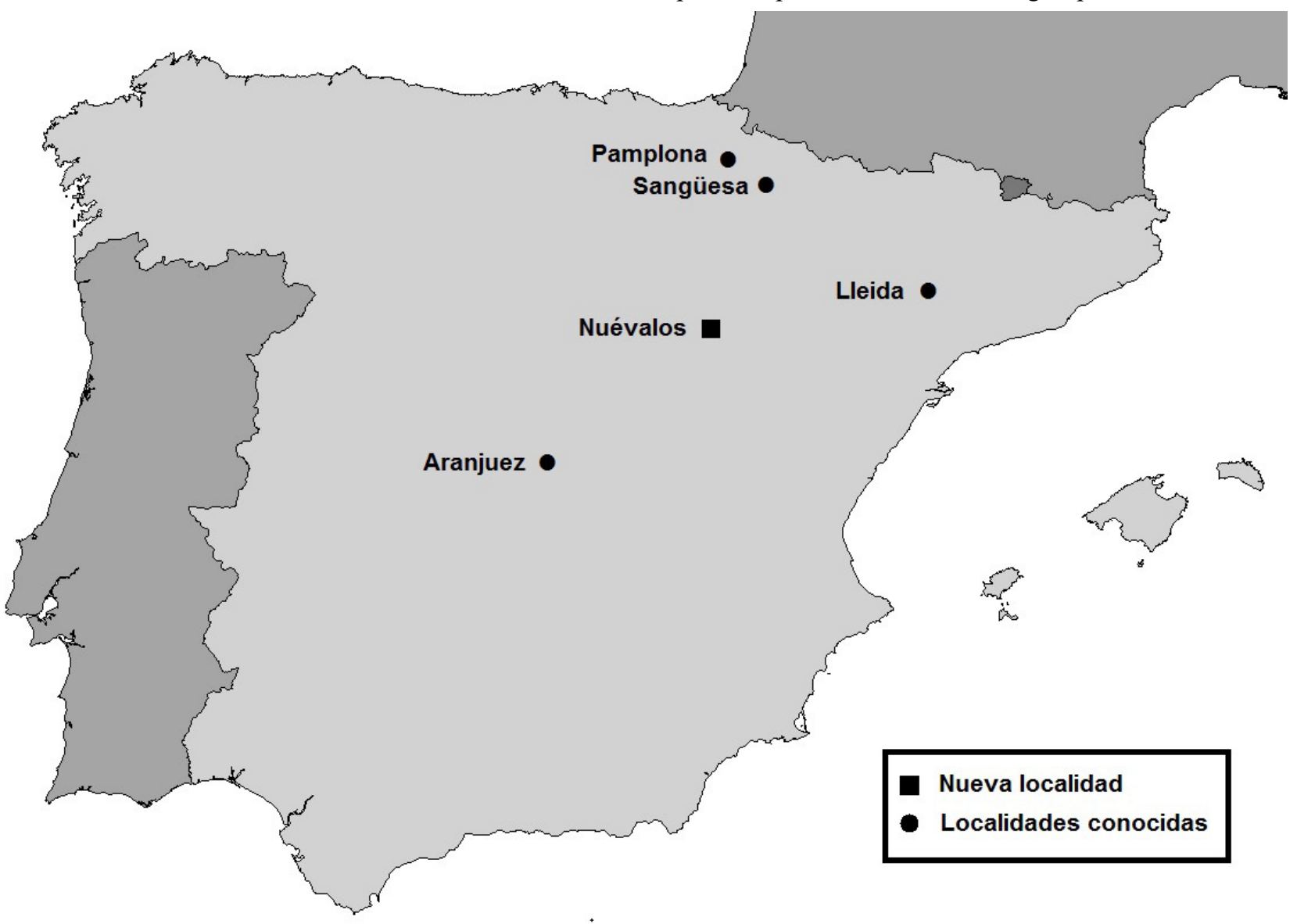

Fig. 1 - Distribución de las localidades con agrupaciones conocidas de Nyctalus noctula en España. 


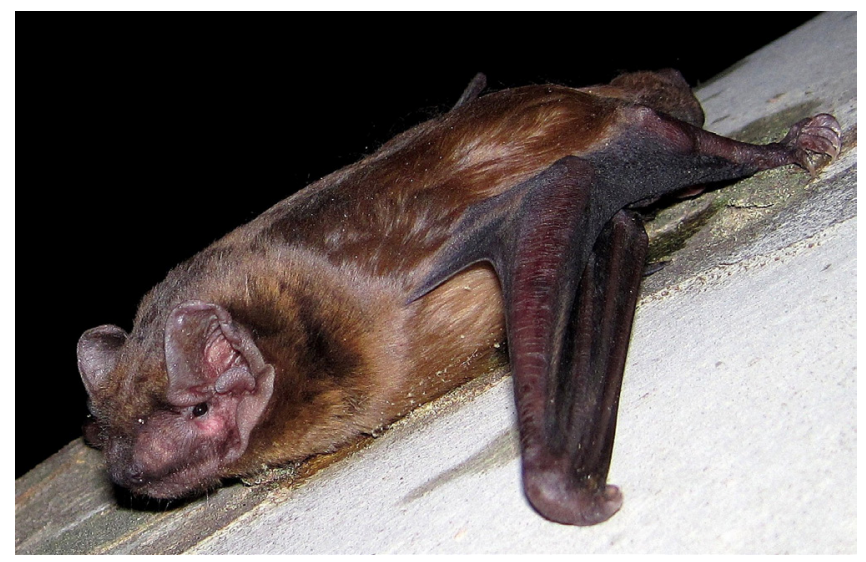

Fig. 2 - Nyctalus noctula.

situados a elevada altura, tal como los que prefiere seleccionar para evitar la depredación (Ruczynski \& Bogdanowicz 2005). En España, todas las agrupaciones conocidas se encuentran en parques urbanos (Ruedi et al. 1998; Alcalde 2007; Camprodon \& Guixé 2013) muy distantes unos de otros. Esto induce a sospechar sobre la escasez de áreas forestales naturales adecuadas que satisfagan sus preferencias.

La conservación del conjunto de arbolado ornamental del Monasterio de Piedra parece asegurada por el momento debido a la aparente buena condición sanitaria de los árboles y a la ausencia de podas y otro tipo de manejo que pudiera afectarles. Un estudio de la composición y tamaño poblacional, y la selección de refugios de $N$. noctula en este enclave permitiría evaluar su importancia y prever alternativas de manejo ante eventuales actuaciones sobre el entorno. Por otra parte sería interesante coordinar el seguimiento que se realizan en las colonias conocidas y comprobar si existe alguna relación entre ellas.

\section{Agradecimientos}

Expresamos nuestro agradecimiento al director del Centro Turístico del Monasterio de Piedra José Pont y al ex director Javier Rodríguez, así como al resto del personal empleado, por la inestimable ayuda prestada durante nuestra estancia. A la Sección de Conservación de la Biodiversidad del Servicio Provincial de Zaragoza (Departamento de Agricultura, Ganadería y Medio Ambiente del Gobierno de Aragón) por la financiación de algunos de los trabajos. A la Estación Biológica de Doñana (CSIC) a través de Javier Juste por la identificación molecular de los individuos capturados y a Juan Tomás Alcalde por la revisión del manuscrito y sus sugerencias. A Juan Ignacio Llana por su ayuda y asistencia en el campo. A los miembros del Equipo de Edición por sus correcciones y sugerencias.

\section{REFERENCIAS}

Alcalde, J. T. 1999. New ecological data on the noctule bat (Nyctalus noctula Schreber, 1774) (Chiroptera, Vespertilionidae) in two towns of Spain. Mammalia, 63: 273-280. DOI: https://dx.doi.org/10.1515/ mamm.1999.63.3.273

Alcalde, J. T. 2007. Nyctalus noctula (Schreber, 1774) In Palomo, L. J., Gisbert, J., Blanco, J. C. (eds.). Atlas y libro rojo de los mamíferos terrestres de España. Dirección General para la Biodiversidad-SECEMSECEMU, Madrid. Pp. 228-232.

AlCALde, J. T. 2008. El nóctulo mediano (Nyctalus noctula) en Pamplona. Colección Biodiversidad Urbana de Pamplona. Ayuntamiento de Pamplona. 52 p.

Camprodon, J. \& Guixé, D. 2013. Estado poblacional, selección de refugios y ecología espacial de las poblaciones de nóctulo grande (Nyctalus lasiopterus) y nóctulo mediano (Nyctalus noctula) en Cataluña. Barbastella 6 (1): 51-59. DOI: http://dx.doi. org/10.14709/BarbJ.6.1.2013.07

Dietz, C. I, Helversen, O. \& Nill, D. 2009. Bats of Britain, Europe \& Northwest Africa. London: A\&C Black Publishers Ltd.

Hutterer. R., Ivanova, T., Meyer-Cords, C. \& Rodrigues, L. 2005. Bat migrations in Europe - A review of banding data and literature. Federal Agency for Nature Conservation, Bonn, 94-96.

IbáÑez, C., Guillén, A., Fernández, R., PÉrez, J. L. \& GUERRERO, S. I. 1992. Iberian distribution of some little known bat species. Mammalia, 56 (3): 433-444. DOI: https://dx.doi.orh/10.1515/mamm.1992.56.3.433

RUCZYNSKI, I. \& BogdAnOwicz, W. 2005. Roost cavity selection by Nyctalus noctula and $N$. leisleri (Vespertilionidae, Chiroptera) in Bialowieza Primeral Forest, eastern Poland. Journal of Mammalogy 86(5): 921-930. DOI: http://dx.doi.org/10.1644/15451542(2005)86[921:RCSBNN]2.0.CO;2

Ruedi, M., Tupinier, Y. \& PAZ, O. DE, 1998. First breeding record for the noctule bat (Nyctalus noctula) in the Iberian Peninsula. Mammalia, 62: 301-304.

Simmons, N.B. 2005. Order Chiroptera. In: Wilson D.E, \& REEDER, D.M. (eds) Mammal species of the world, Vol $1,3^{\text {rd }}$ edn. Johns Hopkins University Press, Baltimore, MD, p 312-529.

Woutersen, K. \& BAfaluy Zoriguel, J. J. 2001. Murciélagos del Alto Aragón. Ed. La Val de Onsera, Huesca. 